ROCZNIKI PSYCHOLOGICZNE/ANNALS OF PSYCHOLOGY

2019, XXII, 1, 53-71

DOI: http://dx.doi.org/10.18290/rpsych.2019.22.1-4

DOROTA SZCZYGIEŁ

WIESŁAW BARYŁA

SWPS University of Social Sciences and Humanities, Faculty in Sopot, Poland

\title{
CHOOSING \\ BETWEEN EMOTION REGULATION STRATEGIES \\ TO APPEAR COOL, CALM, AND COLLECTED: DOES EMOTIONAL STIMULUS INTENSITY MATTER?
}

\begin{abstract}
The present study examined the effect of negative emotional stimulus intensity (low versus high) on the choice of emotion regulation (ER) strategy when a person wants to control their emotional expression, and the impact of this choice on how the information accompanying emotional stimuli is remembered. The effects of emotional stimulus intensity on the choice of ER strategy were examined in two studies. In both studies, the participants (unaware of the differences in the intensity of stimuli) were asked to view images inducing negative emotions of high and low intensity and to choose which strategy (cognitive reappraisal or expressive suppression) they would use in order to control their emotional expression. In addition, in Study 2, the authors tested the memory of the verbal content accompanying the emotional stimuli that appeared during the ER period. As expected, the participants chose reappraisal over suppression when confronted with low-intensity stimuli. In contrast, when confronted with high-intensity stimuli, they chose suppression over reappraisal. The results of Study 2 revealed that memory accuracy was higher for those images that the participants chose to use reappraisal rather than suppression.
\end{abstract}

Keywords: emotion regulation; suppression; reappraisal; choice; intensity; memory.

\section{INTRODUCTION}

Emotions are an essential part of human functioning, as they contribute to adaptive behavior and are well suited for coping successfully with environmental

Corresponding author: DOROTA SZCZYGIEL - SWPS University of Social Sciences and Humanities, Faculty in Sopot, ul. Polna 16/20, 81-745 Sopot, Poland; e-mail: dszczygiel@ swps.edu.pl 
requirements (Frijda, 1986). There are situations, however, when emotions lead to dysfunctional behavior and where the ability to implement emotion regulation (ER) strategies that are contextually appropriate is crucial (Gross \& John, 2003; Webb, Miles, \& Sheeran, 2012). Indeed, an expanding body of research demonstrates that effective management of emotions is associated with better cognitive, emotional, and social functioning (Gross, 2015).

ER is a broad concept that has been defined in many ways (e.g., Gratz \& Roemer, 2004; Gross, 1998; Koole, 2010; Parkinson \& Totterdell, 1999). In this study, we refer to Gross's process model of ER, which is considered one of the most prominent and meaningful models of ER (Webb et al., 2012). In his model, Gross (1998) defines ER as the set of processes by which we influence what emotions we experience, when we experience them, and how we experience and express them. He distinguishes two major classes of ER strategies: antecedent-focused ER and response-focused ER. Antecedent-focused ER acts early in the emotion generation process (i.e., before emotional response tendencies become fully activated to change behavioral and peripheral physiological responding). Response-focused ER refers to the regulatory processes that occur after an emotion has been generated; it involves emotion modification once an emotion has been elicited and once response tendencies have been fully activated. Gross (1998) also distinguishes five different types of ER strategies according to the point at which they impact the emotion-generative process: situation selection, situation modification, attentional deployment, cognitive change, and response modulation.

In the current study, we focus on cognitive reappraisal (hereafter reappraisal) and expressive suppression (hereafter suppression). The former strategy represents antecedent-focused ER, while the latter represents response-focused ER. Reappraisal involves changing the way one thinks about a potentially emotioneliciting situation to alter its emotional impact and, consequently, change one's emotional response to that situation (Gross \& John, 2003). Suppression involves conscious and effortful inhibition of the overt expression of emotion while one is emotionally aroused (Gross \& John, 2003).

\section{Different consequences of reappraisal and suppression}

A growing number of studies, both experimental and correlational (i.e., analyzing individual differences in the use of reappraisal and suppression), show that reappraisal and suppression differ in their consequences. Experimental studies have demonstrated that reappraisal leads to a decrease in both expressive 
behavior and the experience of negative emotion, whereas suppression results in a decrease in behavioral responses but fails to decrease emotional experience (Gross \& Levenson, 1993, 1997). In addition, people who habitually use reappraisal in their daily lives experience and express more positive and less negative emotions, whereas people who use suppression more frequently experience and express less positive and more negative emotions (Gross \& John, 2003; John \& Gross, 2007).

There is also ample evidence demonstrating that suppression impairs cognitive functioning whereas reappraisal leaves cognitive functioning intact (Dillon, Ritchey, Johnson, \& LaBar, 2007; Hayes et al., 2010). There is evidence showing that suppression downgrades the memory of the events that transpired during the period of suppression (Richards, 2004; Richards \& Gross, 1999). Similarly, Richards, Butler, and Gross (2003) demonstrated that, in comparison to reappraisal, the suppression of vocal and facial emotional cues while discussing relationship conflicts with a romantic partner led to worse memory of the actual exchange. Moreover, suppression leads to poorer emotional information processing (Szczygieł, Buczny, \& Bazińska, 2012) and poorer performance on working memory tasks (e.g., Inzlicht \& Gutsell, 2007; Szczygieł \& Maruszewski, 2015).

To fully complement this rather unfavorable picture, it should be said that suppression leads to poorer social functioning (Butler et al., 2003; Gross, 2002, 2015; Verzeletti, Zammuner, Galli, \& Agnoli, 2016). A recent meta-analysis exploring the effect of suppression on social and interpersonal outcomes revealed that suppression was associated with lower social support, poorer quality of social interactions, and, consequently, with poorer social satisfaction (Chervonsky $\&$ Hunt, 2017).

At this point it should be added that although an accumulating body of research shows that expressive suppression can lead to negative short- and long-term consequences, especially when it is excessively (i.e., habitually and inflexibly) used across a variety of situational contexts, expressive suppression can also be adaptive; for example, the ability to hide one's emotions in certain situations can be essential for creating and sustaining positive social relations (e.g., Gross, 2002) and maintaining employment (e.g., Grandey, 2000).

\section{Does emotional intensity matter when choosing emotion regulation strategies?}

The literature reviewed in the previous section provides clear evidence that, compared to reappraisal, suppression is associated with higher costs and lower 
benefits. Interestingly, the results of Loewenstein's (2007) survey showed that the majority of the respondents considered suppression most effective in downregulating negative emotions. Moreover, more than two-thirds of respondents declared that in the face of unpleasant situations (i.e., ones causing negative emotions), they would use suppression ("I will myself to be calm, cool and collected"), whereas just over fifty percent of respondents stated that they would use reappraisal ("Think about the situation from a different perspective"). At first glance, these results are puzzling, as they suggest that people generally prefer using suppression rather than reappraisal. Taking a closer look at Loewenstein's (2007) study, however, we find that this may be a somewhat simplified conclusion. First, the participants were asked to imagine their experiences in very unpleasant situations (e.g., cleaning up vomit at a party, being falsely accused of cheating on an exam). Second, they were asked to imagine that, following this, they would need to control their emotions to appear "in a positive state of mind for someone they were about to meet" (Loewenstein, 2007, p. 189). Thus, the tendency to favor suppression as an ER strategy, as observed in Loewenstein's (2007) study, was revealed in situations triggering intense negative emotions at a time when people wanted to withhold (or at least minimize) their negative emotional responses for social reasons.

Indeed, the results of a study by English, Lee, John, and Gross (2017) demonstrated that, in day-to-day life, suppression was used more frequently when people wanted to control their demeanor - that is, when they were guided by interpersonal motives, such as "to keep up appearances" and "to avoid conflicts." In contrast, reappraisal was used more often when people wanted to control their own feelings - that is, when they were guided by a hedonic motive, such as "to change their mood in order to feel better" (English et al., 2017). Moreover, suppression was used more often when people were in the company of other people, especially with non-close partners, whereas reappraisal use did not differ depending on whether participants were alone or not. It should be noted, however, that in this study, as in Loewenstein's (2007) survey, participants reported their use of ER strategies in relation to the most negative events of the day (English et al., 2017). It is not clear, therefore, whether the intensity (low vs. high) of negative emotional contexts plays a role in shaping people's preferences for using suppression (vs. reappraisal). This is the issue that inspired the current study.

The influence of emotional stimulus intensity, considered a key dimension of variability in situational contexts (Sheppes, Scheibe, Suri, \& Gross, 2011; Sheppes \& Levin, 2013), on ER strategy choice, defined as "the act of indepen- 
dent choice between different regulation strategies that are available in a specific context" (Sheppes \& Levin, 2013, p. 1), has been extensively researched in recent years (Shafir, Schwartz, Blechert, \& Sheppes, 2015; Sheppes, 2014; Sheppes \& Levin, 2013). Studies have focused mainly on cognitive ER strategies: distraction and reappraisal. The results demonstrated that when confronted with low-intensity emotional contexts, people showed a preference for reappraisal (over distraction) but when confronted with high-intensity emotional contexts, people showed a preference for distraction (over reappraisal) (Shafir et al., 2015; Sheppes et al., 2011; Sheppes et al., 2014). It should be noted, however, that in the above-mentioned studies participants were asked to choose an ER strategy that would best help them to decrease their negative emotional experiences. Therefore, we still do not know if emotional stimulus intensity matters when people are motivated to decrease their emotional expression and their available options are reappraisal and suppression.

There is some indirect evidence, from research on emotional labor (EL), suggesting that the choice between suppression and reappraisal may depend on the emotional intensity of the situation. EL focuses on how service employees regulate their emotions in order to match the display rules of the organization (Hochschild, 1983). Hochschild (1983) defines EL as "the management of feeling to create a publicly observable facial and bodily display" (Hochschild, 1983, p. 7) and distinguishes two EL strategies: surface acting (SA) and deep acting (DA). SA refers to modifying emotional displays without changing internal feelings and usually involves suppressing one's felt emotions while faking the desired ones. In contrast, DA refers to efforts aimed at changing the emotion felt in order to elicit the appropriate emotional display.

It has been claimed that although Gross's process model of ER and the concept of EL represent different research traditions, they are aligned at the theoretical level. Both concepts relate to how people regulate their emotions, but the use of EL strategies is related to a specific context (i.e., work ) and to specific motives (i.e., managing emotions in order to comply with expectations for emotional expression within the organization), whereas ER, as defined by Gross, is a broader concept that is universally applicable to any situation and motive (including hedonic and instrumental motives; Tamir, 2016). Grandey (2000) suggested that DA corresponds to reappraisal and that SA corresponds to suppression. Indeed, there is evidence showing that reappraisal is significantly and positively related to DA, whereas suppression is significantly and positively related to SA (Lee et al., 2016). 
There is also evidence to suggest that the propensity to employ SA vs DA is partly determined by the situational context, especially the emotional intensity of the situation. For example, Diefendorff, Richard, and Yang (2008) demonstrated that DA is used in low-stress situations, while SA is applied in high-stress situations. Grandey, Dickter, and Sin (2004) observed that employees reported using DA during mildly stressful interactions with customers, whereas highly stressful interactions were associated with SA. These results suggest that people may switch between reappraisal (an equivalent of DA) and suppression (an equivalent of SA) depending on the intensity of the emotional context.

\section{The current study}

The main purpose of the present study was to investigate the effect of emotional stimulus intensity on the choice between suppression and reappraisal when one wishes to withhold one's emotional expression. We focused on reappraisal and suppression for three reasons. First, both strategies received the most attention in the literature as they are commonly used in everyday life (Gross \& John, 2003; Gross, 2002; John \& Gross, 2004; English et al., 2017). Second, both strategies decrease expressive behavior associated with negative emotions (Gross \& Thompson, 2007; English et al., 2017). Third, as mentioned earlier, both strategies are perceived as effective means to down-regulate negative emotions for social reasons, that is, when people are guided by interpersonal motives while applying ER strategies (Loewenstein, 2007). We predicted that in low-intensity emotional contexts people would be more likely to use reappraisal (vs. suppression) and that in high-intensity emotional contexts they would be more likely to use suppression (vs. reappraisal) (Hypothesis 1).

The second aim of our study was to examine whether the implementation of a chosen ER strategy affects the memory of the events that occurred during the period of regulation. As mentioned before, there is ample evidence showing that, compared to reappraisal, suppression leads to poorer memory functioning (Dillon et al., 2007; Hayes et al., 2010). Notably, this adverse impact of suppression on memory was observed in studies with a between-subjects design, in which participants were instructed to use either suppression or reappraisal (e.g., Webb et al., 2012). To the best of our knowledge, no study has analyzed the impact of suppression versus reappraisal on memory when people autonomously choose between the two. In order to address this research gap, we used a within-subjects design to compare the effects of reappraisal and suppression on memory. Building upon previous results, we hypothesized that, compared to reappraisal, sup- 
pression would worsen the memory of verbal content accompanying emotional stimuli that appeared during the period of regulation (Hypothesis 2).

We tested our hypotheses in two experiments, in which we used a previously validated ER choice paradigm (Sheppes et al., 2011; Sheppes et al., 2014) that allowed us to manipulate the intensity of emotional stimuli by using standardized images evoking negative emotions of high and low intensity. Thus, the structure of our experiments was adapted from Sheppes et al. (2011) as follows: whereas Sheppes et al. asked participants to regulate their emotions in order to feel less negative emotions by using either reappraisal or distraction, in our experiments the participants were asked to regulate their emotions in order to express less negative emotions by using either reappraisal or suppression. The protocol was approved by the Departmental Ethics Committee, SWPS University of Social Sciences and Humanities, Faculty in Sopot (Ref. No. WKE-S-15-IX-42).

\section{STUDY 1}

In Study 1, our goal was to examine which of the two ER strategies (reappraisal or suppression) the participants choose to employ in response to lowintensity and high-intensity negative stimuli.

\section{METHOD}

Participants. A total of 40 (50\% female) full-time and part-time students aged 20 to $55(M=33.50, S D=9.22)$ from the Gdańsk University of Technology voluntarily participated in the study.

Stimuli for the emotion regulation choice task. The stimuli for the ER choice task (ERCT) were derived from the International Affective Picture System (IAPS; Lang, Bradley, \& Cuthbert, 2008) and belonged to a set of images used in previous studies using the ER choice paradigm (e.g., Sheppes et al., 2014). The ERCT consisted of 20 pictures, of which 10 were of low intensity (Arousal: $M=4.90, S D=0.66$; Valence: $M=3.41, S D=0.24$ ) and 10 were of high intensity (Arousal: $M=6.30, S D=0.70$; Valence: $M=1.86, S D=0.31$ ). Valence and arousal differed significantly for low- and high-intensity images (both $F \mathrm{~s}>21.63, p$ s <.001). Previous studies have confirmed that such differences in arousal and valence are sufficient to induce different levels of intensity in emotional responses (Sheppes et al., 2014). The codes for the IAPS images 
used in the low-intensity category are: 2278, 2490, 2691, 6010, 6190, 6836, $7360,9102,9120,9470$. The codes for the IAPS images in the high-intensity category are: 2053, 2800, 3000, 3068, 3140, 3150, 3180, 3261, 3530, 9410. Each trial comprised previewing a picture for one second, choosing either reappraisal or suppression (indefinite time), and then employing the chosen strategy while viewing the picture again for five seconds. Participants indicated their choice by pressing a button on the keyboard and confirming it out loud. Both the order of the pictures and the choice options were counterbalanced across the experimental trials.

Procedure. When the participants arrived at the laboratory, they were given a brief introduction to ER. They were informed that the study would examine ER choice and that their task would be to refrain from expressing emotions while viewing unpleasant images. It was made clear that this goal could be achieved in two ways: either by simply withholding the visible signs of negative emotions (i.e., suppression) or by thinking about the image in a way that would reduce its negative meaning, making it possible to feel and, ultimately, show less negative emotions (i.e., reappraisal). The participants were instructed that they were free to choose either strategy during the task. No one refused to participate. Next, the participants listened to verbal descriptions of how to employ reappraisal and suppression and were provided with concrete examples of the use of each ER strategy (see Bebko, Franconeri, Ochsner, \& Chiao, 2011; Gross, 1998). They were instructed that if they chose suppression they were expected to inhibit facial emotional expressions and any other noticeable signs of emotion, so that someone watching them would not be able to tell what they were feeling. As an example of expressive suppression, the experimenter described a shop assistant who encounters a difficult and unpleasant customer but does not want to show what he feels and simply holds his poker face regardless of the unpleasant circumstances. Furthermore, the participants were instructed that when they chose reappraisal, they were expected to reinterpret the images in ways that decreased their negative meaning. For example, the image of a small crying boy sitting at the edge of a road could initially be interpreted as conveying the sadness of an abandoned child. When reappraising the image to feel less negative, the experimenter suggested that one could imagine that the boy was only tired and was likely to meet his parents soon. After signing the informed consent form, the participants were seated at a $60 \mathrm{~cm}$ viewing distance from a laptop screen. Next, they were trained to regulate their emotional reactions using reappraisal or suppression by viewing four negative images for five seconds and either think about the image in a way that reduced its negative meaning (i.e., reappraisal) or inhibit emotional 
expression (i.e., suppression). This training phase included two low-intensity trials and two high-intensity trials (one reappraisal trial and one suppression trial for each intensity level). The participants then completed four trials in which they viewed four negative images for one second each and were instructed to use suppression or reappraisal when the picture appeared again (for five seconds). In this training phase, the strategies were predetermined (one trial at each intensity level for each strategy). In the subsequent four trials (two trials at each intensity level), the participants were free to choose which strategy they wanted to implement. They were instructed to indicate their choice by pressing a button on the keyboard and confirming it out loud. For the sake of simplicity, the participants were given code names for suppression ("poker face") and reappraisal ("think differently"). They were encouraged to discuss the strategy with the experimenter as stimulus presentation occurred and, when needed, they were corrected. Once the experimenter was sure that the participants understood the difference between suppression and reappraisal and were able to implement both strategies, the choice phase began. After completing the ERCT, the participants were debriefed, thanked for their time, treated to sweets, and invited to watch a short funny movie about animals. The majority of the participants stopped with sweets. They were accompanied by the female experimenter throughout the experiment; they received no remuneration. The codes for the IAPS images used in the training phase are: 2130, 2205, 2590, 2722, 2753, 9041 (low-intensity pictures) and 3010, 3064, 3080, 3101, 3110, 3120 (high-intensity pictures).

\section{RESULTS AND DISCUSSION}

In order to verify our Hypothesis 1, predicting that for low-intensity images participants would show a preference for reappraisal and that for high-intensity images they would show a preference for suppression, we performed a repeated measures analysis of variance with the frequency of reappraisal strategy as the dependent variable. The dependent variable was quantified as the total number of times a participant chose reappraisal divided by the number of images in that condition; this figure was subsequently converted into the reappraisal index, expressed as a percentage. Therefore, the suppression index was the complement of the reappraisal index. As expected, the analysis revealed a significant effect for Intensity. Specifically, participants chose reappraisal more often for low-intensity images $(M=78.5 \%, S D=19.94,95 \%$ CI $[72.12,84.88])$ compared to high-intensity ones $(M=27.00 \%, S D=28.89,95 \%$ CI $[17.44,36.56])$; in addi- 
tion, participants chose suppression more often for high-intensity images $(M=73.00 \%, 95 \%$ CI $[63.44,82.56])$ than for low-intensity ones $(M=21.50 \%$, $95 \%$ CI $[15.12,27.88]), F(1,39)=73.22, p<.001, \eta_{\mathrm{p}}{ }^{2}=.65$. This expected bias in regulatory choices was observed in $72.5 \%$ (29/40) of the participants. These results support Hypothesis 1.

\section{STUDY 2}

The aim of Study 2 was twofold: (1) to replicate the results of Experiment 1; (2) to examine the impact of implementing the freely chosen ER strategy on cognitive functioning - that is, on the memory of verbal content accompanying emotional stimuli during the period of regulation.

\section{Participants and procedure}

A total of 40 (50\% female) full-time and part-time students aged 20 and 63 $(M=35.23, S D=11.25)$ from the SWPS University of Social Sciences and Humanities volunteered to participate in Experiment 2, the course of which was similar to Study 1. Compared to Study 1, we introduced several changes. First, we increased the number of trials in the ERCT. The following IAPS images were added to the set of low-intensity images: 1301, 2312, 2700, 9160, 9440. The set of high-intensity images was supplemented with the following: 3230, 6831, 9181, 9252, 9420. This means that the images used in Experiment 2 were the same as those used in Sheppes et al. (2011). Accordingly, the ERCT consisted of 30 pictures, of which 15 were of low intensity (Arousal: $M=5.01, S D=0.70$; Valence: $M=3.41, S D=0.24$ ) and 15 were of high intensity (Arousal: $M=6.12$, $S D=0.69$; Valence: $M=1.89, S D=0.33)$. Valence and arousal differed significantly for low- and high-intensity images (both $F \mathrm{~s}>19.01, p \mathrm{~s}<.001$ ). Second, each image presented to the participants (during both the training and choice phases) was accompanied by a single-sentence description of its content. The description was placed at the bottom of the image during the five seconds while the selected strategy was being implemented. Third, in order to ensure that the participants actually implemented the chosen strategy, they were asked (after each trial in both the training and choice phases) to confirm which they had just implemented. In the training phase, three participants were not sure whether they actually understood and followed the instructions, leading to repetition. In the choice phase, none of the participants reported more than two failures in the im- 
plementation of the chosen strategy; therefore, data from all study participants were included in the analyses. Fourth, immediately after the choice phase, the participants were given an unexpected memory test to gauge their grasp of the verbal information presented during the period of ER. On each memory test trial, the participants were presented with the same images as in the choice phase, but this time each image was accompanied by two single-sentence descriptions, one of which was slightly different than the one presented in the choice phase. For example, in the choice phase, the image "Riot" (IAPS code 2291) was described as "A man throwing a stone in a police blockade." In the memory test, the participants were presented with two descriptions: "A man throwing a stone in a police blockade" and "A man throwing a stone at a police car." The participant's task was to indicate out loud which of these descriptions had appeared in the choice phase.

\section{RESULTS AND DISCUSSION}

The results show that participants chose to implement reappraisal in $76.83 \%$ ( $S D=17.79 \%)$ of the low-intensity trials (95\% CI: [71.14, 82.53]), and suppression in $75.17 \%(S D=18.05 \%)$ of the high-intensity trials $(95 \% \mathrm{CI}$ : [69.39, $80.94]), F(1,39)=108.05, p<.0001, \eta_{\mathrm{p}}^{2}=.74$. This bias was observed in $82.5 \%$ (33/40) of the participants. These findings replicate the results obtained in Experiment 1 and provide clear support for Hypothesis 1.

In order to verify our Hypothesis 2, predicting that the memory of information collected during the period of regulation would be impaired following the choice of suppression, relative to reappraisal, we performed a repeated measures analysis of variance with memory accuracy as the dependent variable. Memory accuracy was indexed by the number of correct answers, which were subsequently converted into percentages. The participants' performance on the memory test was fair. It was significantly greater than chance $(50 \%)$ both for images viewed under reappraisal, $t(39)=7.89, p<.001$, and for those viewed under suppression, $t(39)=3.39, p=.002$. The analysis revealed that participants' memory was impaired more strongly following suppression $(M=58.33 \%$ correct, $S D=15.55 \%$, 95\% CI: $[53.35,63.30])$ compared to reappraisal $(M=69.97 \%$ correct, $S D=16.00 \%, 95 \%$ CI: [64.85, 75.09]), $F(1,39)=10.96, p=.002, \eta_{\mathrm{p}}{ }^{2}=.22$. These findings show that, compared to reappraisal, suppression weakens the memory of information collected during the period of regulation and, thus, support Hypothesis 2. 


\section{GENERAL DISCUSSION}

The primary goal of the current study was to examine the effect of emotional stimulus intensity on the choice between ER strategies when one needs to withhold their emotional expression. Consistently with our prediction, the participants chose reappraisal over suppression when viewing low-intensity negative images and suppression over reappraisal when viewing high-intensity negative images. These results were revealed in two independent studies. Therefore, our study demonstrates that people control their emotional expression by flexibly switching between ER strategies: they prefer reappraisal (which focuses on emotional experience) when confronted with low-intensity stimuli, but they opt for suppression (which directly focuses on emotional expression) when confronted with high-intensity stimuli.

Our results extend previous research showing that reappraisal is a preferred ER strategy in low-intensity contexts. While previous research demonstrated that reappraisal (rather than distraction) was preferred when people wanted to decrease their negative experience (Sheppes et al., 2011; Sheppes et al., 2014), our study demonstrates that reappraisal (rather than suppression) is also preferred when people want to decrease their negative expression.

Why is controlling emotions through suppression (rather than reappraisal) preferred in a high-intensity context? There is ample empirical evidence to suggest that exposure to stimuli eliciting high arousal consumes the available attentional resources and thus deteriorates information processing (Derakshan \& Eysenck, 2009; Eysenck \& Calvo, 1992; Mather \& Sutherland, 2011). This is particularly important in the case of reappraisal, which requires engagement with the emotionally challenging situation in order to reinterpret its meaning (Sheppes, 2014) and at the same time entails the necessity of overcoming the tendency to identify with this situation and its contents (Shafir et al., 2015). Accordingly, reappraisal requires substantial cognitive effort, especially in high-intensity emotional contexts (Shafir et al., 2015). It is therefore very likely that the implementation of reappraisal is perceived as too difficult (if at all possible) in a high-intensity context because individuals do not have sufficient cognitive resources to generate alternative interpretations of highly emotional situations. In such instances, suppression may be the only option available when one wants to control one's emotional expression. It is also very likely that people choose an ER strategy based on their emotion-related knowledge of which strategy works in a given situational context. This knowledge may be explicit or implicit (Mikolajczak, 2009). In other words, people may simply know, from their previous 
experience, that reappraisal is ineffective in particularly demanding emotional situations (Sheppes, Catran, \& Meiran, 2009). The possible mechanisms underlying the choice between reappraisal and suppression at different intensities should be examined in future studies.

The results of our study contribute to the literature on EL. As mentioned earlier, there is evidence that DA (equivalent to reappraisal) is mainly used in low-stress situations, while SA (equivalent to suppression) is used in high-stress situations (e.g., Grandey et al., 2004). Our study complements these results by showing that, indeed, when a person wishes to control their expressive behavior, the choice between suppression (equivalent to SA) and reappraisal (equivalent to DA) is moderated by the intensity of negative emotional contexts.

The second objective of the current study was to extend previous work on the impact of suppression and reappraisal on how accurately information presented during the regulation period is remembered. As expected, memory accuracy differed significantly as a function of the chosen strategy: memory accuracy was higher when participants chose to use reappraisal over suppression. These results are largely consistent with previous work showing that suppression impairs memory performance (e.g., Dillon et al., 2007; Richards \& Gross, 1999, 2000). It should be noted that in the above-mentioned studies participants were instructed to use either reappraisal or suppression. Our study contributes to the literature by demonstrating that reappraisal is also more beneficial than suppression in regard to memory accuracy when the use of either strategy is the result of free choice.

The literature offers at least two explanations of why suppression, compared to reappraisal, leads to poorer memory performance. First, suppression requires constant self-control and self-correction during an emotional event. This constant self-awareness reduces an individual's cognitive resources, making them less available for processing the information that transpired during the period of suppressing, which results in worse memory. Reappraisal, which is an antecedent-focused strategy, affects the emotion-generative process earlier than suppression and probably does not require continuous self-regulatory effort during an emotional event, as is the case with suppression. Accordingly, reappraisal leaves cognitive resources intact for the processing of events, which are then better remembered (Gross, 2002). The second explanation refers to the levels-of-processing effect (Craik \& Lockhart, 1972), which focuses on the depth of information processing and predicts that the deeper the information is processed, the better and longer it is remembered. Some researchers (e.g., Dillon et al., 2007; Hayes et al., 2010) argue that reappraisal encourages a more meaningful analysis of a negative 
stimulus, promoting elaborative semantic encoding, whereas suppression is focused on inhibiting emotional expression, which distracts attention from the elaboration of the stimulus, resulting in poorer memory performance. It is important to note that these two explanations are not mutually exclusive.

There are several limitations to the current study that suggest directions for future research. First, in both experiments we induced negative emotions by means of affective images, which only symbolically represent real-life events. Therefore, in order to strengthen the validity of the findings further, future research might use other ways to evoke negative emotions, more compatible with real life. Second, only two ER strategies were compared. Although we conducted our research using the ER choice paradigm, participants could only choose suppression or reappraisal (i.e., they were forced to implement one of those two strategies). The limited selection of potentially available strategies limits the scope of our research. The third limitation of our research pertains to Study 1, in which we did not ask the participants whether they actually adhered to the chosen ER strategy (this limitation was overcome in Study 2). We believe that it is important to check whether participants actually use the ER strategy they chose earlier, as research shows that people have a tendency to use multiple ER strategies in response to emotion-eliciting stimuli (Aldao \& Nolen-Hoeksema, 2013). Future research should also verify the effectiveness of the strategies used by participants (Did the participants effectively refrain from emotional expression?). This is particularly important when examining the consequences of ERs, as was the case in this study. We did not verify the effectiveness of the strategies applied by the participants, which should be considered a limitation of Study 2. Fourth, memory assessment was limited only to the verbal content accompanying the stimuli triggering emotions. One of the possible ways of extending research on the impact of ER on memory would be to adopt the procedure used by Sheppes and colleagues (2011), who created a memory test consisting of the images originally presented during the ER strategy implementation and their Photoshop-modified versions. Fifth, it cannot be ruled out that higher memory accuracy for the reappraised images was partly due to the availability of the image descriptions during the implementation of ER strategies. It is likely that when reappraisal was selected as an ER strategy, the image description may have been used as a clue to reinterpret the meaning of the image (i.e., to create a less negative narrative), which in turn may have improved the memory of this description and thus influenced the results of the study. In order to overcome this shortcoming and ambiguity, further research is needed to confirm the results presented here. Finally, our study does not take into account the diversity of personality traits, 
motivational factors, and cognitive abilities, all of which have been recognized as factors underlying the use of specific ER strategies (e.g., Opitz, Lee, Gross, \& Urry, 2014; Sheppes, 2014; Sheppes \& Levin, 2013; Wang, Shi, \& Li, 2009). For this reason, and also taking into account the evidence demonstrating that people differ in the spontaneous use of ER strategies in response to online emotions arising in the present moment (Egloff, Schmukle, Burns, \& Schwerdtfeger, 2006; Ehring, Tuschen-Caffier, Schnülle, Fischer, \& Gross, 2010; Volokhov \& Demaree, 2010), it can be predicted that there are differences between individuals that influence their approach to negative stimuli and determine how they choose to alter the impact of those stimuli on the experienced and expressed emotions. For example, there is evidence that people high in trait emotional intelligence are able to use ER strategies in a more flexible manner than their counterparts low in emotional intelligence because they have access to more ER strategies (PeñaSarrionandia, Mikolajczak, \& Gross, 2015; Szczygieł \& Mikolajczak, 2017). It would, therefore, be desirable to develop more sophisticated research paradigms that could capture the complexity of potential determinants of ER strategy choices better.

The results of our study and those obtained by other researchers using the ER choice paradigm (e.g., Sheppes, 2014) open up new perspectives in clinical psychology research. A growing body of research suggests that rigid (inflexible) use of ER strategies, especially those considered costly and ineffective in altering uncomfortable or unwanted emotions, contributes to depression and anxiety disorders (Campbell-Sills \& Barlow, 2007; Liu \& Thompson, 2017; Moore, Zoellner, \& Mollenholt, 2008). Indeed, according to a recent meta-analysis, people with current and remitted depression have difficulties in using adaptive ER strategies (e.g., reappraisal) and tend to apply maladaptive ER ones (e.g., suppression) (Visted, Vøllestad, Nielsen, \& Schanche, 2018). More importantly, there is evidence showing that depression is linked to a limited ability to choose ER strategies rather than to a limited ability to implement them (Liu \& Thompson, 2017). In other words, individuals suffering from depression are able to deploy various ER strategies when instructed to do so, but in daily life they spontaneously (and habitually) use maladaptive strategies (Ehring et al., 2010; Moore et al., 2008). The results showing that people suffering from depression can make excessive use of suppression when other, more effective strategies can be used instead, have led Moore and colleagues (2008) to conclude that psychological interventions to alleviate depressive symptoms should aim both to broaden the repertoire of ER strategies available to the patient and to enhance the patient's 
ability to make flexible choices between strategies, depending on the situation (see also Campbell-Sills \& Barlow, 2007).

\section{REFERENCES}

Aldao, A., \& Nolen-Hoeksema, S. (2013). One versus many: Capturing the use of multiple emotion regulation strategies in response to an emotion-eliciting stimulus. Cognition \& Emotion, 27(4), 753-760.

Bebko, G. M., Franconeri, S. L., Ochsner, K. N., \& Chiao, J. Y. (2011). Look before you regulate: Differential perceptual strategies underlying expressive suppression and cognitive reappraisal. Emotion, 11(4), 732-742.

Butler, E. A., Egloff, B., Wilhelm, F. H., Smith, N. C., Erickson, E. A., \& Gross, J. J. (2003). The social consequences of expressive suppression. Emotion, 3(1), 48-67.

Campbell-Sills, L., \& Barlow, D. H. (2007). Incorporating emotion regulation into conceptualizations and treatments of anxiety and mood disorders. In J. J. Gross (Ed.), Handbook of emotion regulation (pp. 542-559). New York, NY, US: Guilford Press.

Chervonsky, E., \& Hunt, C. (2017). Suppression and expression of emotion in social and interpersonal outcomes: A meta-analysis. Emotion, 17, 669-683.

Craik, F. I., \& Lockhart, R. S. (1972). Levels of processing: A framework for memory research. Journal of Verbal Learning and Verbal Behavior, 11, 671-684.

Derakshan, N., \& Eysenck, M. W. (2009). Anxiety, processing efficiency, and cognitive performance: New developments from attentional control theory. European Psychologist, 14(2), 168-176.

Diefendorff, J. M., Richard, E. M., \& Yang, J. (2008). Linking emotion regulation strategies to affective events and negative emotions at work. Journal of Vocational Behavior, 73, 498-508.

Dillon, D. G., Ritchey, M., Johnson, B. D., \& LaBar, K. S. (2007). Dissociable effects of conscious emotion regulation strategies on explicit and implicit memory. Emotion, 7, 354-365.

Egloff, B., Schmukle, S. C., Burns, L. R., \& Schwerdtfeger, A. (2006). Spontaneous emotion regulation during evaluated speaking tasks: Associations with negative affect, anxiety expression, memory, and physiological responding. Emotion, 6, 356-366.

Ehring, T., Tuschen-Caffier, B., Schnülle, J., Fischer, S., \& Gross, J. J. (2010). Emotion regulation and vulnerability to depression: Spontaneous versus instructed use of emotion suppression and reappraisal. Emotion, 10, 563-572.

English, T., Lee, I. A., John, O. P., \& Gross, J. J. (2017). Emotion regulation strategy selection in daily life: The role of social context and goals. Motivation and Emotion, 41, 230-242.

Eysenck, M. W., \& Calvo, M. G. (1992). Anxiety and performance: The processing efficiency theory. Cognition \& Emotion, 6(6), 409-434.

Frijda, N. H. (1986). The emotions. Cambridge, UK: Cambridge University Press.

Grandey, A. A. (2000). Emotional regulation in the workplace: A new way to conceptualize emotional labor. Journal of Occupational Health Psychology, 5, 95-110.

Grandey, A. A., Dickter, D. N., \& Sin, H.-P. (2004). The customer is not always right: Customer aggression and emotion regulation of service employee. Journal of Organizational Behavior, $25,397-418$. 
Gratz, K. L., \& Roemer, L. (2004). Multidimensional assessment of emotion regulation and dysregulation: Development, factor structure, and initial validation of the difficulties in emotion regulation scale. Journal of Psychopathology and Behavioral Assessment, 26, 41-54.

Gross, J. J. (1998). Antecedent- and response-focused emotion regulation: Divergent consequences for experience, expression, and physiology. Journal of Personality and Social Psychology, 74, 224-237.

Gross, J. J. (2002). Emotion regulation: Affective, cognitive, and social consequences. Psychophysiology, 39, 281-291.

Gross, J. J. (2015). Emotion regulation: Current status and future prospects. Psychological Inquiry, 26, 1-26.

Gross, J. J., \& John, O. P. (2003). Individual differences in two emotion regulation processes: Implications for affect, relationships, and well-being. Journal of Personality and Social Psychology, 85, 348-362.

Gross, J. J., \& Levenson, R. W. (1993). Emotional suppression: Physiology, self-report, and expressive behavior. Journal of Personality and Social Psychology, 64, 970-986.

Gross, J. J., \& Levenson, R. W. (1997). Hiding feelings: The acute effects of inhibiting negative and positive emotion. Journal of Abnormal Psychology, 106(1), 95-103.

Gross, J. J., \& Thompson, R. A. (2007). Emotion regulation: Conceptual foundations. In J. J. Gross (Ed.), Handbook of emotion regulation (pp. 3-24). New York, NY, US: Guilford Press.

Hayes, J. P., Morey, R. A., Petty, C. M., Seth, S., Smoski, M. J., McCarthy, G., \& LaBar, K. S. (2010). Staying cool when things get hot: Emotion regulation modulates neural mechanisms of memory encoding. Frontiers in Human Neuroscience, 4, 230. DOI: 10.3389/fnhum. 2010.00230

Hochschild, A. R. (1983). The managed heart: Commercialization of human feeling. Berkeley, CA, US: University of California Press.

Inzlicht, M., \& Gutsell, J. N. (2007). Running on empty: Neural signals for self-control failure. Psychological Science, 18, 933-937.

John, O. P., \& Gross, J. J. (2004). Healthy and unhealthy emotion regulation: Personality processes, individual differences, and life span development. Journal of Personality, 72(6), 1301-1334.

John, O. P., \& Gross, J. J. (2007). Individual differences in emotion regulation strategies: Links to global trait, dynamic, and social cognitive constructs. In J. J. Gross (Ed.), Handbook of emotion regulation (pp. 351-372). New York, NY, US: Guilford Press.

Koole, S. L. (2010). The psychology of emotion regulation: An integrative review. Cognition \& Emotion, 23(1), 4-41.

Lang, P. J., Bradley, B. N., \& Cuthbert, B. N. (2008). International Affective Picture System (IAPS): Affective ratings of pictures and instruction manual [Technical Rep. A-8]. Gainesville, FL, US: University of Florida.

Lee, M., Pekrun, R., Taxer, J. L., Schutz, P. A., Vogl, E., \& Xie, X. (2016). Teachers' emotions and emotion management: Integrating emotion regulation theory with emotional labor research. Social Psychology of Education, 19, 843-863.

Liu, D. Y., \& Thompson, R. J. (2017). Selection and implementation of emotion regulation strategies in major depressive disorder: An integrative review. Clinical Psychology Review, 57, 183-194.

Loewenstein, G. (2007). Affective regulation and affective forecasting. In J. J. Gross (Ed.), Handbook of emotion regulation (pp. 180-203). New York, NY, US: Guilford Press. 
Mather, M., \& Sutherland, M. R. (2011). Arousal-biased competition in perception and memory. Perspectives on Psychological Science, 6(2), 114-133.

Mikolajczak, M. (2009). Going beyond the ability-trait debate: The three-level model of emotional intelligence. Electronic Journal of Applied Psychology, 5, 25-31.

Moore, S. A., Zoellner, L. A., \& Mollenholt, N. (2008). Are expressive suppression and cognitive reappraisal associated with stress-related symptoms? Behaviour Research and Therapy, 46, 993-1000.

Opitz, P. C., Lee, I. A., Gross, J. J., \& Urry, H. L. (2014). Fluid cognitive ability is a resource for successful emotion regulation in older and younger adults. Frontiers in Psychology, 5, 609. DOI: $10.3389 /$ fpsyg.2014.00609

Parkinson, B., \& Totterdell, P. (1999). Classifying affect-regulation strategies. Cognition \& Emotion, 13(3), 277-303.

Peña-Sarrionandia, A., Mikolajczak, M., \& Gross, J. J. (2015). Integrating emotion regulation and emotional intelligence traditions: A meta-analysis. Frontiers in Psychology, 6, 160. DOI: 10.3389/fpsyg.2015.00160

Richards, J. M. (2004). The cognitive consequences of concealing feelings. Current Directions in Psychological Science, 13, 131-134.

Richards, J. M., Butler, E. A., \& Gross, J. J. (2003). Emotion regulation in romantic relationships: The cognitive consequences of concealing feelings. Journal of Social and Personal Relationships, 20(5), 599-620.

Richards, J. M., \& Gross, J. J. (1999). Composure at any cost? The cognitive consequences of emotion suppression. Personality and Social Psychology Bulletin, 25, 1033-1044.

Richards, J. M., \& Gross, J. J. (2000). Emotion regulation and memory: The cognitive costs of keeping one's cool. Journal of Personality and Social Psychology, 79, 410-424.

Shafir, R., Schwartz, N., Blechert, J., \& Sheppes, G. (2015). Emotional intensity influences preimplementation and implementation of distraction and reappraisal. Social Cognitive and Affective Neuroscience, 10(10), 1329-1337.

Sheppes, G. (2014). Emotion regulation choice: Theory and findings. In J. J. Gross (Ed.), Handbook of emotion regulation ( $2^{\text {nd }}$ ed., pp. 126-139). New York, NY, US: Guilford Press.

Sheppes, G., Catran, E., \& Meiran, N. (2009). Reappraisal (but not distraction) is going to make you sweat: Physiological evidence for self-control effort. International Journal of Psychophysiology, 71(2), 91-96.

Sheppes, G., \& Levin, Z. (2013). Emotion regulation choice: Selecting between cognitive regulation strategies to control emotion. Frontiers in Human Neuroscience, 7, 179. DOI: 10.3389/fnhum.2013.00179

Sheppes, G., Scheibe, S., Suri, G., \& Gross, J. J. (2011). Emotion-regulation choice. Psychological Science, 22, 1391-1396.

Sheppes, G., Scheibe, S., Suri, G., Radu, P., Blechert, J., \& Gross, J. J. (2014). Emotion regulation choice: A conceptual framework and supporting evidence. Journal of Experimental Psychology: General, 143, 163-181.

Szczygieł, D., Buczny, J., \& Bazińska, R. (2012). Emotion regulation and emotional information processing: The moderating effect of emotional awareness. Personality and Individual Differences, 52, 433-437.

Szczygieł, D., \& Maruszewski, T. (2015). Why expressive suppression does not pay? Cognitive costs of negative emotion suppression: The mediating role of subjective tense-arousal. Polish Psychological Bulletin, 46, 336-349. 
Szczygiel, D., \& Mikolajczak, M. (2017). Why are people high in emotional intelligence happier? They make the most of their positive emotions. Personality and Individual Differences, 117, 177-181.

Tamir, M. (2016). Why do people regulate their emotions? A taxonomy of motives in emotion regulation. Personality and Social Psychology Review, 20(3), 199-222.

Verzeletti, C., Zammuner, V. L., Galli, C., \& Agnoli, S. (2016). Emotion regulation strategies and psychosocial well-being in adolescence. Cogent Psychology, 3(1), 1199294. DOI: 10.1080/ 23311908.2016.1199294

Visted, E. V., Vøllestad, J. J., Nielsen, M. M., \& Schanche, E. E. (2018). Emotion regulation in current and remitted depression: A systematic review and meta-analysis. Frontiers in Psychology, 9, 756. DOI: 10.3389/fpsyg.2018.00756

Volokhov, R. N., \& Demaree, H. A. (2010). Spontaneous emotion regulation to positive and negative stimuli. Brain and Cognition, 73(1), 1-6.

Wang, L., Shi, Z., \& Li, H. (2009). Neuroticism, extraversion, emotion regulation, negative affect and positive affect: The mediating roles of reappraisal and suppression. Social Behavior and Personality, 37(2), 193-194.

Webb, T. L., Miles, E., \& Sheeran, P. (2012). Dealing with feeling: A meta-analysis of the effectiveness of strategies derived from the process model of emotion regulation. Psychological Bulletin, 138(4), 775-808. 